\title{
Effect of non-ideal power take-off efficiency on performance of single- and two-body reactively controlled wave energy converters
}

\author{
António F. O. Falcão ${ }^{1}$ • João C. C. Henriques ${ }^{1}$
}

Received: 10 November 2014 / Accepted: 16 March 2015 / Published online: 10 April 2015

(c) Springer International Publishing AG 2015

\begin{abstract}
The highest efficiency of wave energy capture by point absorbers is attained under conditions close to resonance. In most cases, the natural frequency of resonance is higher than the typical frequency of the waves. Reactive phase control has been proposed to improve these situations. Reactive power contributes nothing to the average delivered power and is back-and-forth exchange of energy between the power take-off system (PTO) and the oscillating system. Apart from larger peaks in PTO forces and power, a major drawback of reactive phase control is the energy loss by dissipative processes inherent to the back-and-forth energy exchange, especially if the magnitude of such exchanged energy is comparable to, or even significantly larger than, the net absorbed energy, which may be the case in point absorbers. The paper considers a floating axisymmetric twobody wave energy converter constrained to oscillate in heave in deep water. The outer body is a buoy, whereas the inner body is a long surface-piercing cylinder against whose inertia the outer floater reacts. The PTO converts the relative motion between the two bodies and the associated forces into useful energy. The basic equations in the frequency domain for the reactively controlled two-body converter performance in regular waves are presented for an imperfectly efficient PTO. If the inner cylindrical body is fixed, the equations are reduced to a single-degree-of-freedom. The analysis is extended to irregular waves characterized by a variance density spectrum. Numerical results are presented for regular and irregular waves, including reactive control optimization.
\end{abstract}

António F. O. Falcão

antonio.falcao@tecnico.ulisboa.pt

1 LAETA, IDMEC, Instituto Superior Técnico, Universidade de Lisboa, 1049-001 Lisbon, Portugal
Keywords Wave energy - Two-body converter . Hydrodynamics · Reactive control · Optimization

\section{List of symbols}

\section{Roman letters}

$\begin{array}{ll}a & \text { Outer radius of body } 1 \\ A_{i j} & \text { Added mass } \\ A_{\mathrm{W}} & \text { Regular wave amplitude } \\ b & \text { Radius of body } 2 \\ B_{i j} & \text { Radiation damping coefficient } \\ C & \text { Damping coefficient of PTO } \\ d & \text { Draught of body } 2 \\ f & \text { Frequency } \\ f & \text { (With subscript) force } \\ F & \text { (With subscript) complex amplitude of force } \\ g & f \\ G=K /(\omega C) & \text { PTO reactance-to-resistance ratio } \\ H_{\mathrm{S}} & \text { Significant wave height } \\ K & \text { Stiffness of PTO } \\ L & \text { Capture width } \\ P & \text { Power absorbed by PTO } \\ P_{\text {max }} & \text { Theoretical maximum power } \\ P_{\text {out }} & \text { Power output of PTO } \\ P_{\text {wave }} & \text { Wave power } \\ S_{f}(f) & \text { Variance density spectrum } \\ S_{i} & \text { Water-plane area of body } i \\ t & \text { Time } \\ T & \text { Regular wave period } \\ T_{\mathrm{e}} & \text { Complex amplitude of } x_{i} \\ x_{i} & \end{array}$




\section{Greek letters}

$\begin{array}{ll}\Gamma & \text { Excitation force coefficient } \\ \varepsilon & \text { Relative error in Appendix 2 } \\ \eta & \text { PTO efficiency } \\ \rho & \text { Water density } \\ \omega=2 \pi f & \text { Radian frequency }\end{array}$

\section{Subscript}

irr Irregular waves

max Maximum

out Output from PTO

\section{Superscripts}

(1) Unit incident wave amplitude

overbar Time average

asterisk Dimensionless quantity

\section{Introduction}

A wide variety of concepts has been proposed and studied for wave energy conversion. In most cases, the devices are of oscillating body or oscillating water column (OWC) types (Falnes 2007; Falcão 2010; López et al. 2013). The highest efficiency of wave energy absorption by these wave energy converters is attained under conditions close to resonance. Especially in the case of relatively small devices (the so-called point absorbers whose horizontal dimensions are much smaller than the representative wavelength), it is well known that the resonance bandwidth is relatively narrow, which implies that their performance in irregular waves is relatively poor. Besides, for many point absorbers, the natural frequency of resonance is higher than the typical frequency of the waves. Phase control has been proposed to improve these situations (Salter et al. 1976; Falnes and Budal 1978; Falnes 2002a). Reactive phase control is a way of doing that: reactive power contributes nothing to the average delivered power and is back-and-forth exchange of energy between the power take-off system (PTO) and the oscillating system. This energy may be stored in a flywheel, a gas accumulator, a battery of condensers, or may be supplied by the electrical grid. A major drawback of reactive phase control is the energy loss by dissipative processes inherent to the backand-forth energy exchange, especially if the magnitude of such exchanged energy is comparable to, or even significantly larger than, the net absorbed energy. This may be the case of point absorbers. Reactive phase control is particularly appropriate if the PTO is a high-pressure hydraulic circuit with gas accumulator, where the fluid flow may be reversed by controlling the valve system. This may also be achieved in the case of direct electrical energy conversion (linear or rotating generator) by two-way exchange of energy with the grid. Reactive control has also been considered for OWCs equipped with self-rectifying air turbines. If the setting angle of the rotor blades of a Wells turbine is controllable within a sufficiently wide range ( say $\pm 20^{\circ}$ ), then the machine can operate either as a turbine or as a compressor, and may be used to achieve reactive control (Gato and Falcão 1989; Sarmento et al. 1990; Gato et al. 1991). A $400 \mathrm{~kW}$ variablepitch Wells turbine, whose sophisticated control mechanism was driven by eddy currents, was built to be tested in the Pico plant, Azores, Portugal (Taylor and Caldwell 1998), but was never installed. The relatively modest efficiency of the Wells turbine, especially when operating in the compressor mode, severely limits the gains from reactive control (Perdigão and Sarmento 2003). This, in addition to the much higher mechanical complexity of the turbine and inherent reliability problems, has deterred the use of reactive control in OWCs.

An alternative to reactive phase control is control by latching, which avoids the two-way energy transfer and the associated energy dissipation that characterize a reactive phase-controlled PTO. This was first proposed for single oscillating bodies reacting against a fixed reference frame (in general the sea bottom) (Falnes and Budal 1978), and consists in latching the body in a fixed position during certain intervals of the oscillation cycle.

In principle, reactive control may be optimal, i.e. may allow the theoretical maximum wave energy capture, as predicted by linear wave theory, to be achieved in unconstrained amplitude conditions. (such optimal control is non-causal). This is not the case of control by latching, that is necessarily suboptimal (Falnes 2002b, p. 204).

The theory of the energy absorption from regular waves by a single-degree-of-freedom oscillating body with a linear PTO consisting of a linear damper and linear a spring can be found in the pioneer work by Evans (1976), and appeared subsequently in many other papers, including the extension to several degrees of freedom [see also Falnes (2002a, b)]. Korde (2003) studied a system of coupled heaving bodies, comparing devices using a submerged reaction mass with devices where such a mass is on board. The optimal reactive and resistive loads and corresponding wave energy absorption were determined by a two-degree-of-freedom frequency domain model based on regular waves.

Not many papers were published on how reactive control should be designed to take into account the imperfect efficiency of the PTO. The implications of the PTO efficiency on control strategies of heaving point absorbers were addressed by Tedeschi et al. (2011) and Ricci et al. (2011). The maximization of the time-averaged power output of a reactively controlled wave energy converter equipped with an 
imperfectly efficient PTO was performed for the multi-point absorber Wavestar wave energy converter equipped with a hydraulic PTO. A Wavestar point absorber is a buoy fixed at the extremity of a pivoting arm (Hansen and Kramer 2011). Results from raw measurements of generated power from a prototype in the sea were reported in Vidal Sánchez et al. (2012). The performance of a reactively controlled single point-absorber of a Wavestar wave energy converter with a non-ideally efficient PTO was analysed theoretically by Strager et al. (2014) for regular waves, and by Vidal Sánchez et al. (2014) for regular and irregular waves. Genest et al. (2014) proposed partial reactive control as a causal suboptimal control method for a heaving single-body wave energy converter in regular and irregular waves and investigated how it should be designed to account for the less-than-ideal efficiency of the actuators. A general conclusion from these investigations is that the reactive component of the exchanged power should decrease with decreasing efficiency of the PTO actuators.

In the present paper, we consider a floating axisymmetric two-body wave energy converter constrained to oscillate in heave in deep water. The outer body is a floater, whereas the inner floating body is a long surface-piercing cylinder against whose inertia the outer floater reacts. The PTO converts the relative motion between the two bodies and the associated forces into useful energy. The draught of the inner body is assumed large so that the wave energy absorption results essentially from the interaction between the incoming waves and the oscillating outer body. This two-body system is an idealized version of the Powerbuoy and Wavebob wave energy converters (Falcão 2010; Weber et al. 2009). If the inner body is fixed to the sea bottom (possibly tightmoored), then we have the simple case of a single heaving body sliding along a fixed cylinder. This may be regarded as an idealized version of the L-10 buoy equipped with a linear electrical generator, developed at Oregon State University, USA (Elwood et al. 2009). Our theoretical investigation, in which regular and irregular waves are considered, is based on linear water wave theory and the frequency domain analysis.

The basic equations in the frequency domain for the reactively controlled two-body converter performance in regular waves are presented in Sect. 2.1. If the inner cylindrical body is fixed, the equations are reduced to a single-degree-offreedom (Sect. 2.2), which is basically the situation addressed in Strager et al. (2014) for a different type of device. In Sect. 3 , the analysis is extended to irregular waves characterized by a variance density spectrum. Numerical results are presented in Sect. 4 for regular and irregular waves, including reactive control optimization. Throughout the paper, the impedance of the PTO is assumed time-invariant (the control is causal). No optimal or suboptimal phase control in irregular waves is attempted on a wave-to-wave time-scale.

\section{Basic equations for regular waves}

\subsection{Two-body heaving converter}

We consider, as a typical point absorber, a floating axisymmetric two-body wave energy converter constrained to oscillate in heave in deep water (Fig. 1). The outer body 1 is of cylindrical shape, with a conical bottom. The inner body 2 is a long floating cylinder with a flat bottom. The gap between bodies 1 and 2 is assumed small and the friction force between bodies is neglected. The PTO is driven by the relative motion between bodies 1 and 2 . The analysis is based on linear water wave theory, which assumes the amplitudes of the waves and of the body displacements to be small, and neglects real-fluid effects due to viscosity, turbulence and vortex shedding. No constraints are applied to the motion amplitude of the bodies.

Let $x_{1}$ and $x_{2}$ be vertical coordinates defining the position of bodies 1 and 2, respectively, with $x_{1}=x_{2}=0$ in the absence of waves, and $x_{1}, x_{2}$ increasing in the upward direction.

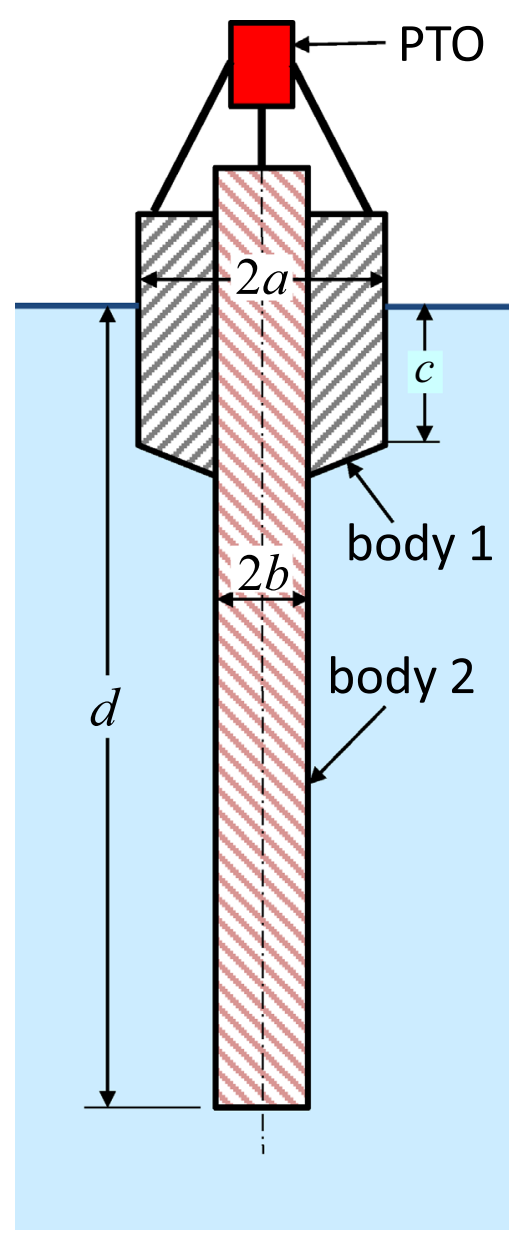

Fig. 1 Schematic representation of the two-body wave energy converter 
We neglect the mass of the PTO system in comparison with the mass $m_{1}$ of body 1 and the mass $m_{2}$ of body 2 , and assume the force $f_{\text {PTO }}$ of the PTO on body 1 to be a linear function of the relative displacement $x_{1}-x_{2}$ and of the relative velocity $\dot{x}_{1}-\dot{x}_{2}$. We write

$f_{\mathrm{PTO}}=-K\left(x_{1}-x_{2}\right)-C\left(\dot{x}_{1}-\dot{x}_{2}\right)$,

where $K$ and $C$ are constant and $C$ is positive. This linear representation, that is introduced to allow a frequency domain analysis to be employed, could be a relatively rough approximation to some real PTO mechanisms. The instantaneous power absorbed by the PTO is

$P(t)=-f_{\mathrm{PTO}}\left(\dot{x}_{1}-\dot{x}_{2}\right)=C\left(\dot{x}_{1}-\dot{x}_{2}\right)^{2}+K\left(x_{1}-x_{2}\right)\left(\dot{x}_{1}-\dot{x}_{2}\right)$.

Assuming linear water wave theory to apply, the governing equations for the dynamics and hydrodynamics of the twobody system may be written as [see e.g. Falnes (2002b), p. 212]

$m_{1} \frac{\mathrm{d}^{2} x_{1}}{\mathrm{~d} t^{2}}=f_{e, 1}+f_{r, 11}+f_{r, 12}-g \rho S_{1} x_{1}+f_{\mathrm{PTO}}$,

$m_{2} \frac{\mathrm{d}^{2} x_{2}}{\mathrm{~d} t^{2}}=f_{e, 2}+f_{r, 22}+f_{r, 21}-g \rho S_{2} x_{2}-f_{\mathrm{PTO}}$.

Here, $f_{e, i}$ is the excitation force on body $i(i=1,2)$ due to the incident waves, $S_{i}$ is the water-plane area of body $i, f_{r, i i}$ is the radiation force on body $i$ due to its own motion, and $f_{r, i j}$ is the radiation force on body $i$ due to the motion of body $j$.

If the waves are regular of radian frequency $\omega$ and amplitude $A_{\mathrm{w}}$, and taking into account that the PTO is linear, we may write, after the transient effects due to initial conditions have died out,

$\left\{x_{i}, f_{e, i}, f_{r, i j}\right\}=\operatorname{Re}\left[\left\{X_{i}, F_{e, i}, F_{r, i j}\right\} \mathrm{e}^{i \omega t}\right]$,

where $X_{i}, F_{e, i}, F_{r, i j}(i, j=1,2)$ are, in general complex, amplitudes and $\operatorname{Re}[]$ stand for real part of. As usual, we decompose the radiation force coefficient $F_{r, i j}$ as

$F_{r, i j}=\left(\omega^{2} A_{i j}-i \omega B_{i j}\right) X_{j} \quad(i, j=1,2)$,

where $A_{i j}$ and $B_{i j}$ are real coefficients of added mass and radiation damping, respectively. It can be shown (Falnes 2002b) that the cross coefficients are equal: $A_{12}=A_{21}, B_{12}$ $=B_{21}$. In the frequency domain, Eqs. (3) and (4) become

$$
\begin{gathered}
\left\{-\omega^{2}\left(m_{1}+A_{11}\right)+i \omega\left(B_{11}+C\right)+\left(g \rho S_{1}+K\right)\right\} X_{1} \\
+\left\{-\omega^{2} A_{12}+i \omega\left(B_{12}-C\right)-K\right\} X_{2}=F_{e, 1}
\end{gathered}
$$

$$
\begin{gathered}
\left\{-\omega^{2}\left(m_{2}+A_{22}\right)+i \omega\left(B_{22}+C\right)+\left(g \rho S_{2}+K\right)\right\} X_{2} \\
+\left\{-\omega^{2} A_{12}+i \omega\left(B_{12}-C\right)-K\right\} X_{1}=F_{e, 2} .
\end{gathered}
$$

We may regard $K / \omega$ and $C$ as the reactive and resistive parts of the PTO impedance.

We assume that the draught $d$ of body 2 is large so that, in the range of frequencies $\omega$ of interest, we may neglect the radiation force $f_{r, 12}$ on body 1 in comparison with $f_{r, 11}$, and neglect the radiation force $f_{r, 21}$ in comparison with the other forces on body 2. On what concerns force $f_{r, 22}$, we only keep its inertial part represented in the frequency domain by $-\omega^{2} A_{22} X_{2}$. Besides, the added mass $A_{22}$ is assumed independent of wave frequency $\omega$ and equal to the added mass of a semi-infinite circular cylinder of radius $b$ oscillating along its own axis in an infinite medium. This value was computed with the aid of WAMIT and is $A_{22}=0.6897 \rho \pi b^{3}$. The mass of the inner cylindrical body 2 is $m_{2}=\rho \pi b^{2} d$. Since the system is axisymmetric, Haskind relationship allows us to write in deep water (Falnes 2002b, p. 149)

$$
B_{11}=\frac{\omega^{3} \Gamma_{1}}{2 \rho g^{3}}
$$

where $\Gamma_{1}=\left|F_{e, 1}\right| / A_{\mathrm{w}}$ is an excitation force coefficient and $A_{\mathrm{w}}$ is incident wave amplitude.

With the assumptions introduced above, Eqs. (7) and (8) become

$$
\begin{aligned}
& \left\{-\omega^{2}\left(m_{1}+A_{11}\right)+i \omega\left(B_{11}+C\right)+\left(g \rho S_{1}+K\right)\right\} X_{1} \\
& \quad-\{i \omega C+K\} X_{2}=F_{e, 1}, \\
& \left\{-\omega^{2}\left(m_{2}+A_{22}\right)+i \omega C+\left(g \rho S_{2}+K\right)\right\} X_{2} \\
& \quad-\{i \omega C+K\} X_{1}=0 .
\end{aligned}
$$

Expressions for the complex amplitudes $X_{1}$ and $X_{2}$ are easily obtained from Eqs. (10) and (11). The time-averaged power absorbed by the PTO, which is equal to the time-averaged power absorbed from the waves, is

$\bar{P}=\frac{1}{2} C \omega^{2}\left|X_{1}-X_{2}\right|^{2}$.

Except if the PTO is perfectly efficient, the time-averaged power output $\bar{P}_{\text {out }}$ is less than the absorbed power $\bar{P}$. The instantaneous power absorbed by the PTO may be written as

$$
P(t)=C\left(\dot{x}_{1}-\dot{x}_{2}\right)^{2}+K\left(x_{1}-x_{2}\right)\left(\dot{x}_{1}-\dot{x}_{2}\right)
$$

or

$$
\begin{aligned}
P(t)= & C \omega^{2}\left|X_{1}-X_{2}\right|^{2} \sin (\omega t+\alpha) \\
& \times[\sin (\omega t+\alpha)+G \cos (\omega t+\alpha)],
\end{aligned}
$$


where $\alpha=\arg \left(X_{1}-X_{2}\right)$, and $G=K /(\omega C)$ is a ratio that may be regarded as a measure of reactiveness. Except if $G=0$, the absorbed power $P(t)$ oscillates between negative and positive values, which characterizes reactive control.

Reactive control implies a two-way energy flux between, on the one side, the oscillating body or bodies and, on the other side, some kind of energy storage system that may be a flywheel, a gas accumulator, a battery of condensers or the electrical grid. Inevitably, there are losses in this energy flux process that may be hydraulic, mechanical, electrical, aerodynamic or of other type. Such losses are to be subtracted from the energy supplied by the wave energy absorber to the PTO whenever $P(t)>0$, and added, when $P(t)<0$, to the energy supplied by the PTO to the oscillating body. We denote by $P_{\text {out }}(t)$ the power output from the PTO and define the PTO efficiency as $\eta_{1}(t)=P_{\text {out }}(t) / P(t)$ if $P(t)>0$, and $\eta_{2}(t)=P(t) / P_{\text {out }}(t)$ if $P(t)<0$, with $\eta_{1}, \eta_{2} \leq 1$. In what follows, we assume that $\eta_{1}=\eta_{2}=\eta$ and that $\eta$ is timeinvariant. Expressions for the time average $\bar{P}_{\text {out }}$ of $P_{\text {out }}(t)$ are given in Appendix 1.

Figure 2 shows a plot of the PTO average efficiency $\bar{\eta}_{\text {PTO }}=\bar{P}_{\text {out }} / \bar{P}$ versus $|G|$, for several values of the efficiency $\eta$. The curves show that, for constant efficiency $\eta$, the average PTO efficiency $\bar{\eta}_{\text {PTO }}$ decreases with increasing $|G|$, the more markedly the smaller the value of $\eta$. The average power output $\bar{P}_{\text {out }}$ is negative if the efficiency $\eta$ is low enough and the ratio $|G|$ is large enough. This observation may have motivated latching phase control to be proposed in the late 1970s.

\subsection{Single-body heaving converter}

We consider now body 2 to be fixed to the sea bottom, with $x_{2}=0$, so that the device may be considered as a singlebody converter. As mentioned above, this may be regarded as an idealized version of the L-10 buoy equipped with a

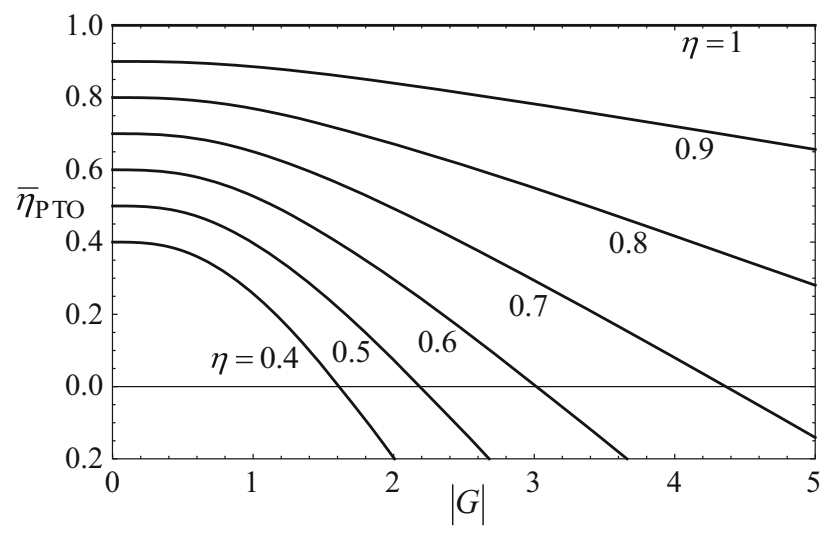

Fig. 2 Average efficiency of PTO, $\bar{\eta}_{\text {PTO }}=\bar{P}_{\text {out }} / \bar{P}$, versus reactiveness ratio $|G|$, for several values of the efficiencies $\eta_{1}=\eta_{2}=\eta$ linear electrical generator (Elwood et al. 2009), in which the inner cylindrical body is tight-moored to the sea bottom. In this case, Eq. (7) reduces to

$$
\left\{-\omega^{2}\left(m_{1}+A_{11}\right)+i \omega\left(B_{11}+C\right)+\left(\rho g S_{1}+K\right)\right\} X_{1}=F_{e, 1},
$$

The time-averaged power absorbed by the PTO, which (in the absence of any dissipative real-fluid effects) is equal to the time-averaged power absorbed from the waves is $\bar{P}=$ $\frac{1}{2} C \omega^{2}\left|X_{1}\right|^{2}$. This may be written as (Evans 1980)

$\bar{P}=\frac{1}{8 B_{11}}\left|F_{e, 1}\right|^{2}-\frac{B_{11}}{2}\left|i \omega X_{1}-\frac{F_{e, 1}}{2 B_{11}}\right|^{2}$.

For given incident wave frequency and amplitude (i.e. for fixed $B_{11}$ and $F_{e, 1}$ ), the condition for maximum $\bar{P}$ is $F_{e, 1}=$ $i 2 B_{11} \omega X_{1}$, which, taking into account Eq. (15), is equivalent, in terms of real quantities, to

$\omega=\sqrt{\frac{\rho g S_{1}+K}{m_{1}+A_{11}}}$,

$C=B_{11}$.

These conditions are well known [see e.g. Falnes (2002b)]. Equation (17) is a resonance condition, whereas Eq. (18) means that the PTO damping should be equal to the radiation damping. If these conditions are satisfied, then we may write, for the maximum time-averaged absorbed power,

$\bar{P}_{\max }=\frac{1}{8 B_{11}}\left|F_{e, 1}\right|^{2}$.

For an axisymmetric (single- or two-body) converter oscillating in heave in deep water, it is (Falnes 2002b, p. 218)

$\bar{P}_{\max }=\frac{\rho g^{3} A_{\mathrm{w}}^{2}}{4 \omega^{3}}$.

\section{Irregular waves}

It may be interesting to investigate the performance of the device in irregular waves with a given variance density spectrum $S_{f}(f)$, as defined in Holthuijsen (2008, p. 36), rather than for a single wave frequency. Here, $f=\omega / 2 \pi$ is wave frequency. The significant wave height and the energy period are given, respectively, by $H_{\mathrm{s}}=4 \sqrt{m_{0}}$ and $T_{\mathrm{e}}=m_{-1} / m_{0}$, where $m_{n}$ is the $n$ th-order moment

$m_{n}=\int_{0}^{\infty}(f)^{n} S_{f}(f) \mathrm{d} f$. 
We consider now irregular waves defined as the superposition of a set of $N$ regular waves of frequency $\omega_{i}$ and amplitude $A_{\mathrm{w}, i}, i=1$ to $N$. The instantaneous power $P_{\text {irr }}(t)$ absorbed from these irregular waves is given by the right-hand side of Eq. (13) with

$x_{1}-x_{2}=\sum_{i=1}^{N} A_{\mathrm{w}, i}\left|X_{1, i}^{(1)}-X_{2, i}^{(1)}\right| \sin \left(\omega_{i} t+\beta_{i}\right)$,

where superscript (1) means that the complex motion amplitudes $X_{1, i}^{(1)}$ and $X_{2, i}^{(1)}$ of bodies 1 and 2 are to be computed for an incident wave of unit amplitude and frequency $\omega_{i}$. In Eq. (22), $\beta_{i}=\arg \left(X_{1, i}^{(1)}-X_{2, i}^{(1)}\right)+\alpha_{i}$, and $\alpha_{i}$ is a random phase in the interval $(0,2 \pi)$. We may write

$$
P_{\text {irr }}(t)=C\left(\dot{x}_{1}-\dot{x}_{2}\right)^{2}+K\left(x_{1}-x_{2}\right)\left(\dot{x}_{1}-\dot{x}_{2}\right) .
$$

Because Eq. (23) involves sums of products of sinusoidal functions, it is

$P_{\mathrm{irr}}(t) \neq \sum_{i=1}^{N} P_{i}(t)$

where $P_{i}(t)$ concerns harmonic of order $i$.

We are particularly interested in time-averaged values. From the orthogonal properties of the sine and cosine functions, we may write

$$
\begin{aligned}
& \lim _{\Delta t \rightarrow \infty} \frac{1}{\Delta t} \int_{0}^{\Delta t} \sum_{i=1}^{N} \sum_{j=1}^{N} Z_{i} Z_{j} \sin \left(\omega_{i} t+\beta_{i}\right) \\
& \times \sin \left(\omega_{j} t+\beta_{j}\right) \mathrm{d} t=\frac{1}{2} \sum_{i=1}^{N} Z_{i}^{2}, \\
& \lim _{\Delta t \rightarrow \infty} \frac{1}{\Delta t} \int_{0}^{\Delta t} \sum_{i=1}^{N} \sum_{j=1}^{N} Z_{i} Z_{j} \sin \left(\omega_{i} t+\beta_{i}\right) \\
& \times \cos \left(\omega_{j} t+\beta_{j}\right) \mathrm{d} t=0,
\end{aligned}
$$

where $Z_{i}$ are constants, and $\omega_{i} \neq \omega_{j}$ if $i \neq j$. It follows that

$\bar{P}_{\text {irr }}(t)=\sum_{i=1}^{N} \bar{P}_{i}$

where an overbar denotes time average. If we replace the superposition of $N$ regular waves by a continuous spectrum, we obtain, from the definition of variance density spectrum $S_{f}(f)$,

$\bar{P}_{\text {irr }}\left(H_{\mathrm{s}}, T_{\mathrm{e}}\right)=2 \int_{0}^{\infty} \bar{P}^{(1)}(2 \pi f) S_{f}(f) \mathrm{d} f$.
Here $\bar{P}^{(1)}(\omega)$ is the time-averaged power absorbed from regular waves of frequency $\omega$ and unit amplitude.

We consider now the power output $P_{\text {irr,out }}(t)$ from the PTO. We write $P_{\text {irr,out }}(t)=\Phi(t) P_{\text {irr }}(t)$, where $\Phi(t)=\eta$ if $P_{\text {irr }}(t) \geq 0$ and $\Phi(t)=\eta^{-1}$ if $P_{\text {irr }}(t)<0$. We recall that $P_{\text {irr }}(t)$ is given by Eqs. (22) and (23). Because of the multiplication by $\Phi(t)$, the orthogonality of the sinusoidal functions no longer applies in the integration with respect to time, and so

$\bar{P}_{\text {out }, \text { irr }}\left(H_{\mathrm{s}}, T_{\mathrm{e}}\right) \neq 2 \int_{0}^{\infty} \bar{P}_{\text {out }}^{(1)}(2 \pi f) S_{f}(f) \mathrm{d} f$,

except if $\eta=1$ (which gives $\Phi=1$ ) or $K=0$ (in which case $P(t)$ is non-negative and $\Phi=\eta$ all the time). In Eq. (29), $\bar{P}_{\text {out }}^{(1)}(\omega)$ is the time-averaged power output in waves of frequency $\omega$ and unit amplitude.

To avoid numerical time-integrations over very long time intervals, we replace inequality (29) by an approximate equality

$\bar{P}_{\text {out }, \text { irr }}\left(H_{\mathrm{s}}, T_{\mathrm{e}}\right) \cong 2 \int_{0}^{\infty} \bar{P}_{\text {out }}^{(1)}(2 \pi f) S_{f}(f) \mathrm{d} f$

and use this approximate equation in the optimization process of searching the values of $C_{e}^{*}$ and $K^{*}$ that maximize $\bar{P}_{\text {out,irr }}$. Here $C_{e}^{*}=C / B_{11}\left(2 \pi T_{\mathrm{e}}\right)$ is defined taking as reference the energy period $T_{\mathrm{e}}$. As before, $B_{11}(\omega)$ is a radiation damping coefficient. The errors introduced using approximation (30) are numerically investigated in Appendix 2.

For an axisymmetric (single- or two-body) converter oscillating in heave in deep water, the theoretical maximum time-averaged power absorbed from irregular waves is

$\bar{P}_{\text {max }, \text { irr }}=2 \int_{0}^{\infty} \bar{P}_{\max }^{(1)}(2 \pi f) S_{f}(f) \mathrm{d} f$,

where $\bar{P}_{\max }^{(1)}(\omega)$ is the theoretical maximum value of the time-averaged power absorbed from regular waves of radian frequency $\omega=2 \pi f$ and unit amplitude. It is given by Eq. (20) with $A_{\mathrm{w}}=1$.

We define a dimensionless time-averaged power output as

$\bar{P}_{\text {out,irr }}^{*}=\frac{\bar{P}_{\text {out,irr }}}{\bar{P}_{\text {max }, \text { irr }}}$.

Another way of characterizing the power performance of the device is through a capture width (defined here in terms of power output)

$L=\frac{\bar{P}_{\text {out,irr }}\left(H_{\mathrm{s}}, T_{\mathrm{e}}\right)}{\bar{P}_{\text {wave }}\left(H_{\mathrm{s}}, T_{\mathrm{e}}\right)}$, 
where

$\bar{P}_{\text {wave }}=\frac{\rho g^{2}}{64 \pi} H_{\mathrm{s}}^{2} T_{\mathrm{e}}$

is the energy flux of the waves per unit crest length in deep water [see McKay (2012)]. We define a dimensionless capture width as $L^{*}=L /(2 a)$, where $2 a$ is the diameter of the outer body.

We adopt a Pierson-Moskowitz variance density spectrum (Goda 2002, p. 28) that may be written in terms of $H_{\mathrm{S}}$ and $T_{\mathrm{e}}$ as

$$
S_{f}(f)=0.1688 H_{\mathrm{s}}^{2} T_{\mathrm{e}}^{-4} f^{-5} \exp \left[-0.675\left(T_{\mathrm{e}} f\right)^{-4}\right] .
$$

The integration in Eq. (31) may be performed analytically and we obtain

$\bar{P}_{\text {max }, \text { irr }}=155.5 \times 10^{-6} \rho g^{3} H_{\mathrm{s}}^{2} T_{\mathrm{e}}^{3}$.

We easily find that $L^{*}$ is related to $\bar{P}_{\text {out,irr }}^{*}$ by

$L^{*}=0.01563 T_{\mathrm{e}}^{* 2} P_{\mathrm{out}, \mathrm{irr}}^{*}$

where

$T_{\mathrm{e}}^{*}=T_{\mathrm{e}}\left(\frac{g}{a}\right)^{1 / 2}$

is a dimensionless energy period.

\section{Numerical results}

We consider now results for the performance of the point absorber shown in Fig. 1, for which $a=c, b=0.4 a$. and the semi-angle of the conical bottom (angle between the generatrices and the axis) is equal to $60^{\circ}$. The volume of the submerged part of body 1 in calm water is $3.031 a^{3}$. The water-plane area is $S_{1}=\pi\left(a^{2}-b^{2}\right)$. Dimensionless values of the added mass $A_{11}$ and radiation damping coefficient $B_{11}$ of body 1 are defined as

$A_{11}^{*}=\frac{A_{11}}{\rho \pi a^{3}}, \quad B_{11}^{*}=\frac{B_{11}}{\rho \pi a^{3} \omega}$.

They were computed with the aid of the boundary-element method WAMIT and are plotted versus dimensionless wave period $T^{*}=2 \pi \omega^{-1} \sqrt{g / a}=T \sqrt{g / a}$ in Fig. 3. Note that $T^{*}$ is numerically equal to the wave period $T=2 \pi \omega^{-1}$ (in seconds) if $g=9.8 \mathrm{~ms}^{-2}$ and $a=9.8 \mathrm{~m}$.

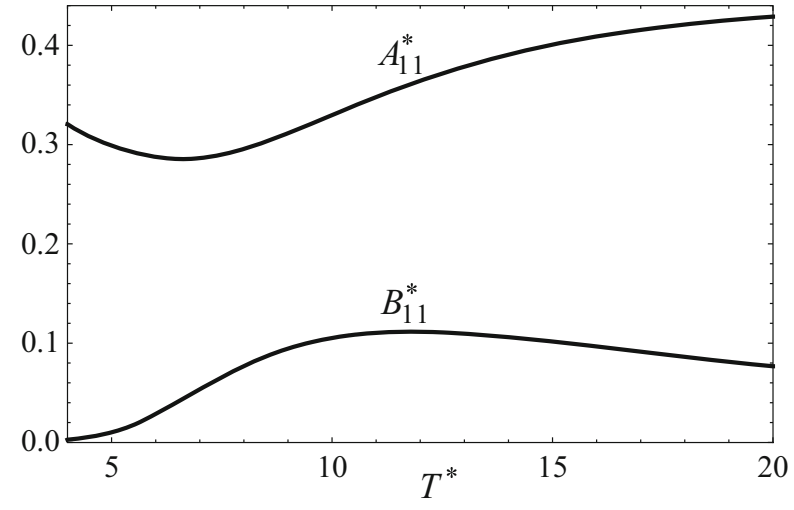

Fig. 3 Dimensionless plot of added mass $A_{11}^{*}$ and radiation damping coefficient $B_{11}^{*}$ versus wave period $T^{*}$, for body 1

\subsection{Single-body-heaving converter in regular waves}

We consider first the case when the inner body 2 is fixed.

The dimensionless period at resonance, for $K=0$, can be obtained from Eq. (17) and is $T^{*}=7.80$.

A dimensionless plot is shown in Fig. 4, with the power output $\bar{P}_{\text {out }}^{*}=\bar{P}_{\text {out }} / \bar{P}_{\text {max }}$ from the PTO versus the wave period $T^{*}$, for several values of the PTO coefficients $C^{*}=$ $C / B_{11}$ and $K^{*}=K\left(\rho g S_{1}\right)^{-1}$, and different values of the PTO efficiencies $\eta_{1}=\eta_{2}=\eta=1.0,0.9,0.8,0.7,0.6$. In the four graphs on the first column of Fig. $4, K^{*}=0$ (pure damping, no reactive power), and it is simply $\bar{P}_{\text {out }}=\eta \bar{P}$; in each graph, the different curves are obtained from the top one by multiplying the ordinates by $\eta$. In the three top graphs of Fig. $4, C^{*}=1$ and so condition (18) $C=B_{11}$ is satisfied. Then the theoretical maximum power output (for an axisymmetric heaving device) is reached (i.e. $\bar{P}_{\text {out }}^{*}=\bar{P}_{\text {out }} / \bar{P}_{\text {max }}=$ 1) if $K$ satisfies the resonance condition (17) and $\eta=1$; this occurs for $T^{*}=7.68$ if $K^{*}=0, T^{*}=8.94$ if $K^{*}=-0.25$, and $T^{*}=11.12$ if $K^{*}=-0.5$. In the four graphs on the third column of Fig. $4, K^{*}=-0.5$ and the reactiveness is relatively high, which allows the floater to be tuned to waves of relatively large period. On the other hand, the curves show that the power output is strongly reduced as the PTO efficiency decreases from unity to 0.6 . This reduction may be attenuated by increasing the damping coefficient $C^{*}$. The same can be said, to a lesser extent, of the four graphs on the centre column of Fig. 4 for which it is $K^{*}=-0.25$.

Figure 5 shows the optimized values (that maximize $\bar{P}_{\text {out }}^{*}$ ) of $K^{*}$ and $C^{*}$ plotted versus wave period $T^{*}$ for the same five values $\eta=1.0,0.9,0.8,0.7,0.6$. The values of $\bar{P}_{\text {out }}^{*}$ and $\left|X^{*}\right|=|X| / A_{\mathrm{w}}$ are also plotted in the figure. The optimization was performed with the aid of the subroutine FindMaximum of Mathematica. If $\eta=1$, then $\bar{P}_{\text {out }}=\bar{P}$, and the optimal values of $C$ and $K$ are given by Eqs. (17) and (18); in particular it is $C=B_{11}$ or $C^{*}=1$. 

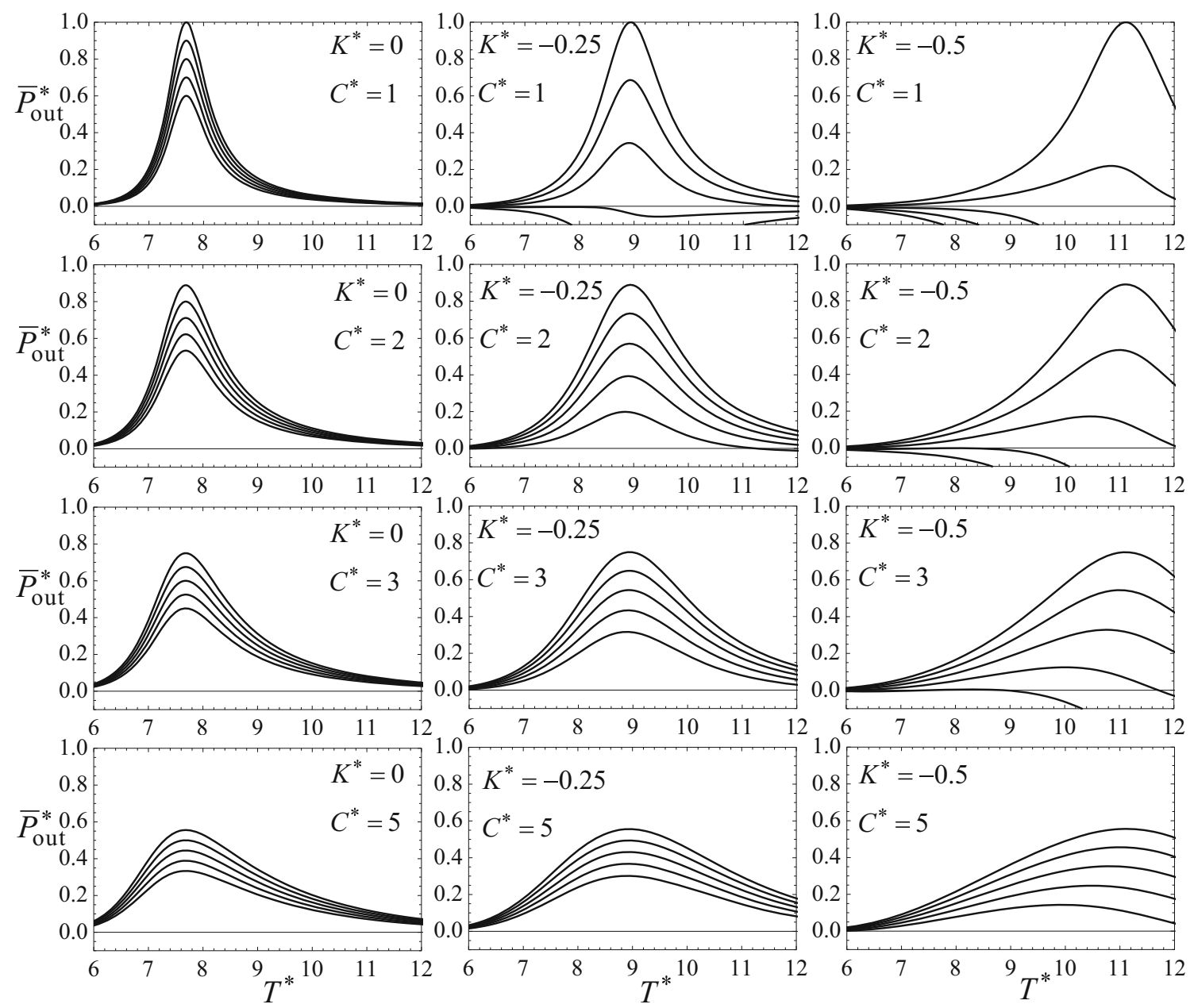

Fig. 4 Dimensionless plot of power output $\bar{P}_{\text {out }}^{*}=\bar{P}_{\text {out }} / \bar{P}_{\text {max }}$ from the PTO versus wave period $T^{*}$ for several values of the PTO parameters $C^{*}=C / B_{11}$ and $K^{*}=K\left(\rho g S_{1}\right)^{-1}$. For each set of curves, it is, from top to bottom, $\eta=1.0,0.9,0.8,0.7,0.6$

For $T^{*}=7.68$, which is the dimensionless value of $T=$ $2 \pi / \omega$ satisfying the resonance condition (17) with $K=0$, the five curves of optimized $K^{*}$ versus $T^{*}$ cross each other at zero ordinate, whereas the five curves of optimized $C^{*}$ versus $T^{*}$ are tangent to each other, the same happening with the five curves of $\left|X^{*}\right|$. These coincidences may be easily explained. We note first that, if $K=0$, we have pure active power, and $\bar{P}_{\text {out }}$ becomes simply equal to the time-averaged power absorbed from the waves, $\bar{P}$, times $\eta$. Hence, if, for some $T$, a pair $(K=0, C)$ maximizes $\bar{P}$, then the same pair of values also maximizes $\bar{P}_{\text {out }}$ independently of the efficiency $\eta$. Obviously, if $K, C, T$ and wave amplitude $A_{\mathrm{w}}$ are fixed, the same is true for motion amplitude $|X|$, independently of the value of $\eta$.

For fixed $T^{*} \neq 7.68$, the optimal value of $C^{*}$ increases with decreasing efficiency $\eta$, the increase being much more dramatic than the changes in optimized $K^{*}$. Not surprisingly, for fixed $T^{*}$, the dimensionless power output $\bar{P}_{\text {out }}^{*}$ decreases with decreasing efficiency $\eta$. For fixed $\eta<1, \bar{P}_{\text {out }}^{*}$ is maximum for $T^{*}=7.68$, that is, for optimal $K$ equal to zero. This is related to the fact that $K=0$, i.e. $G=0$, maximizes the PTO time-averaged efficiency $\bar{P}_{\text {out }}$ (see Fig. 2). For fixed $T^{*} \neq 7.68$, the dimensionless motion amplitude $\left|X^{*}\right|$ decreases with $\eta$, which could be explained by the sharp rise in the optimal damping $C^{*}$.

\subsection{One- and two-body heaving converters in irregular waves}

Numerical results were obtained for three values of the dimensionless draught $d^{*}=d / a=4,6,8$, which roughly cover the range of what could be expected for devices of this type. Results were also computed for the case when body 2 is fixed, which, from the hydrodynamic point of view, is equivalent to the limiting case when the draught $d^{*}$ is infinitely large (the inertia of body 2 increases to infinity). Three different 

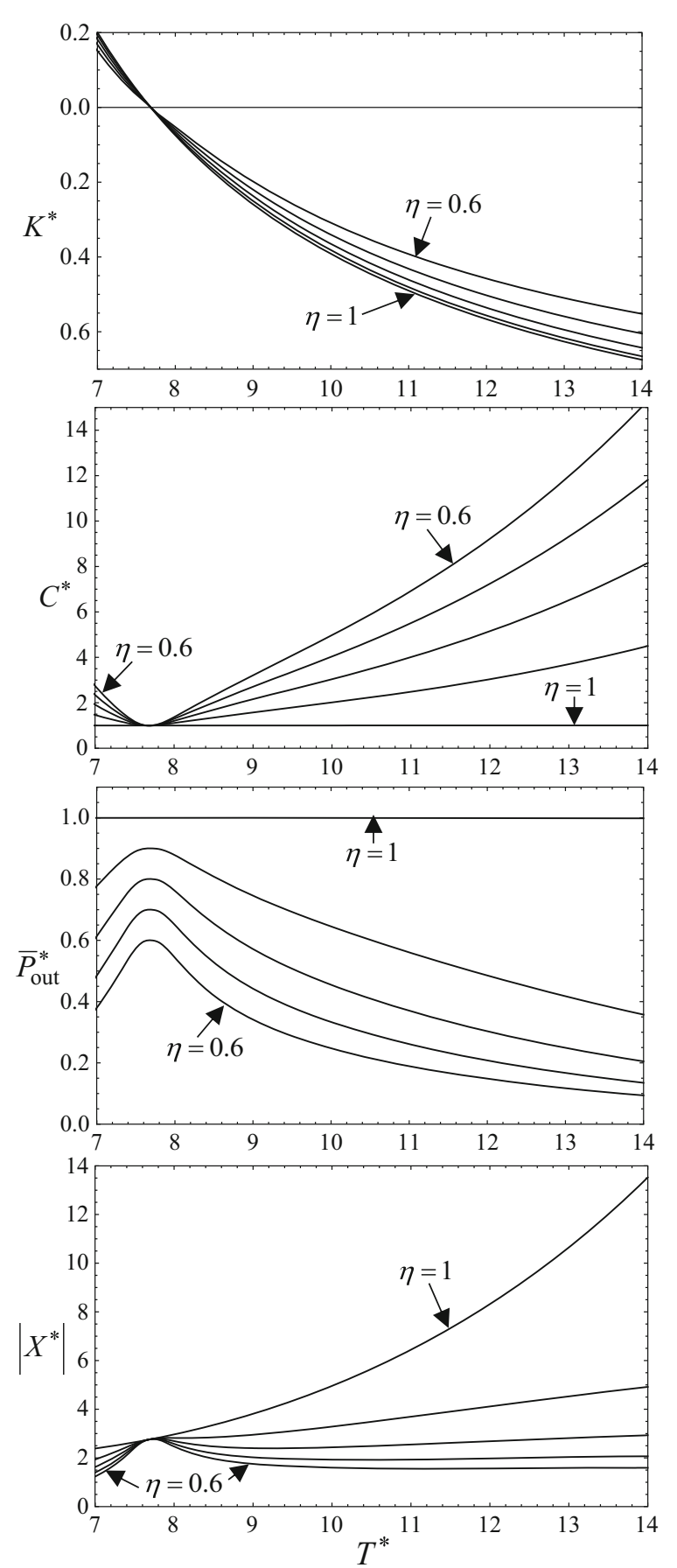

Fig. 5 Optimized PTO parameters $K^{*}$ and $C^{*}$, and corresponding values of $\bar{P}_{\text {out }}^{*}$ and $\left|X^{*}\right|=|X| / A_{\mathrm{w}}$, versus wave period $T^{*}$, for $\eta=1,0.9,0.8,0.7,0.6$

values of the PTO efficiency were considered: $\eta=1.0$ (perfectly efficient PTO), $\eta=0.8$ (a fairly good one) and $\eta=0.6$ (a relatively poor one).

For the numerical evaluation of the integral in Eq. (30), a new variable of integration, $\theta=\left(T_{\mathrm{e}} f\right)^{-1}$ was introduced for convenience. We find

$$
\begin{aligned}
\bar{P}_{\text {out,irr }}\left(H_{\mathrm{s}}, T_{\mathrm{e}}\right)= & 2 \times 0.1688 H_{\mathrm{s}}^{2} \int_{0}^{\infty} \bar{P}_{\text {out }}^{(1)}\left(\frac{2 \pi}{T_{\mathrm{e}} \theta}\right) \theta^{3} \\
& \times \exp \left(-0.675 \theta^{4}\right) \mathrm{d} \theta .
\end{aligned}
$$

The integration was performed in the interval $0.1 \leq \theta \leq 2.0$, discretized into 100 equal segments (the contribution to the integral outside that interval was found to be negligible).

The values for the pair $\left(C_{e}^{*}, K^{*}\right)$ that maximize the dimensionless power output $\bar{P}_{\text {out,irr }}^{*}$ were computed for the $4 \times 3=$ 12 combinations of $d^{*}$ (including a single oscillating body or $d^{*}=\infty$ ) and $\eta$. The subroutine FindMaximum of Mathematica was used in the optimization process. Note that the dimensionless damping coefficient is defined here as $C_{e}^{*}=C / B_{11}\left(\omega_{e}\right)$, where $\omega_{e}=2 \pi / T_{\mathrm{e}}$. The optimized values of $C_{e}^{*}$ and $K^{*}$, together with the maximized values of $\bar{P}_{\text {out,irr }}^{*}$, are plotted in Fig. 6 versus the dimensionless energy period in the range $6 \leq T_{\mathrm{e}}^{*} \leq 12$. Each of the 12 graphs shows three curves for PTO efficiency $\eta=1.0,0.8$ and 0.6 .

We note that the ratio $\bar{P}_{\text {out,irr }}^{*}=\bar{P}_{\text {out, irr }} / \bar{P}_{\text {max, irr }}$ is a measure of the performance quality of the device. A value $\bar{P}_{\text {out, irr }}^{*}=1$ would require a perfectly efficient PTO $(\eta=1)$ providing perfect tuning on a wave-to-wave basis. It is well known that this requires prediction of the incoming waves, apart to relatively heavy computing to be performed in real time [see e.g. Falnes (2002a)]. Besides that is also incompatible with constant values of $K$ and $C$ for a given sea state (i.e. a given spectral distribution). Wave-by-wave phase control is outside the scope of this investigation.

We recall that $T_{\mathrm{e}}^{*}=T_{\mathrm{e}}(g / a)^{1 / 2}$ and so, for economic reasons, the device should be designed for a "large" value of $T_{\mathrm{e}}^{*}$ (a "small" buoy radius $a$ is desirable), subject to the constraint of the overall power performance (represented here by $\bar{P}_{\text {out,irr }}^{*}$ ) not becoming unacceptably low.

Figure 6 shows that the highest value of $\bar{P}_{\text {out,irr }}^{*}$ (about 0.462 ) occurs for the single heaving body and $\eta=1.0$, $T_{\mathrm{e}}^{*}=9.4$. The corresponding values for the PTO stiffness and damping are $K^{*}=-0.56$ and $C_{e}^{*}=2.02$. This implies large reactive power, which is highly penalizing if the PTO efficiency is markedly lower than unity. For more realistic PTO efficiencies and still for the single heaving body, we find, for $\eta=0.8$, a maximum value $\bar{P}_{\text {out,irr }}^{*}=0.30$ at $T_{\mathrm{e}}^{*}=7.2$, and, for $\eta=0.6$, we obtain $\bar{P}_{\text {out,irr }}^{*}=0.21$ at $T_{\mathrm{e}}^{*}=6.8$. The reduction in PTO efficiency $\eta$ is doubly penalizing: the maximum of $\bar{P}_{\text {out,irr }}^{*}$ is severely lowered and occurs for lower values of $T_{\mathrm{e}}^{*}$, i.e. requires larger floater diameters.

It is interesting to note that, for the more realistic cases of $\eta=0.8$ and 0.6 , the curves $\bar{P}_{\text {out, irr }}^{*}$ versus $T_{\mathrm{e}}^{*}$ (third column in Fig. 6) are not very markedly affected by variations in the draught $d^{*}$ (especially in the range $6 \leq T_{\mathrm{e}}^{*} \leq 8$ ). The peaks occur for $T_{\mathrm{e}}^{*} \cong 7$ and take values $\bar{P}_{\text {out,irr }}^{*} \cong 0.28$ to 0.30 for $\eta=0.8$, and $\bar{P}_{\text {out,irr }}^{*} \cong 0.21$ to 0.22 for $\eta=0.6$. The graphs 

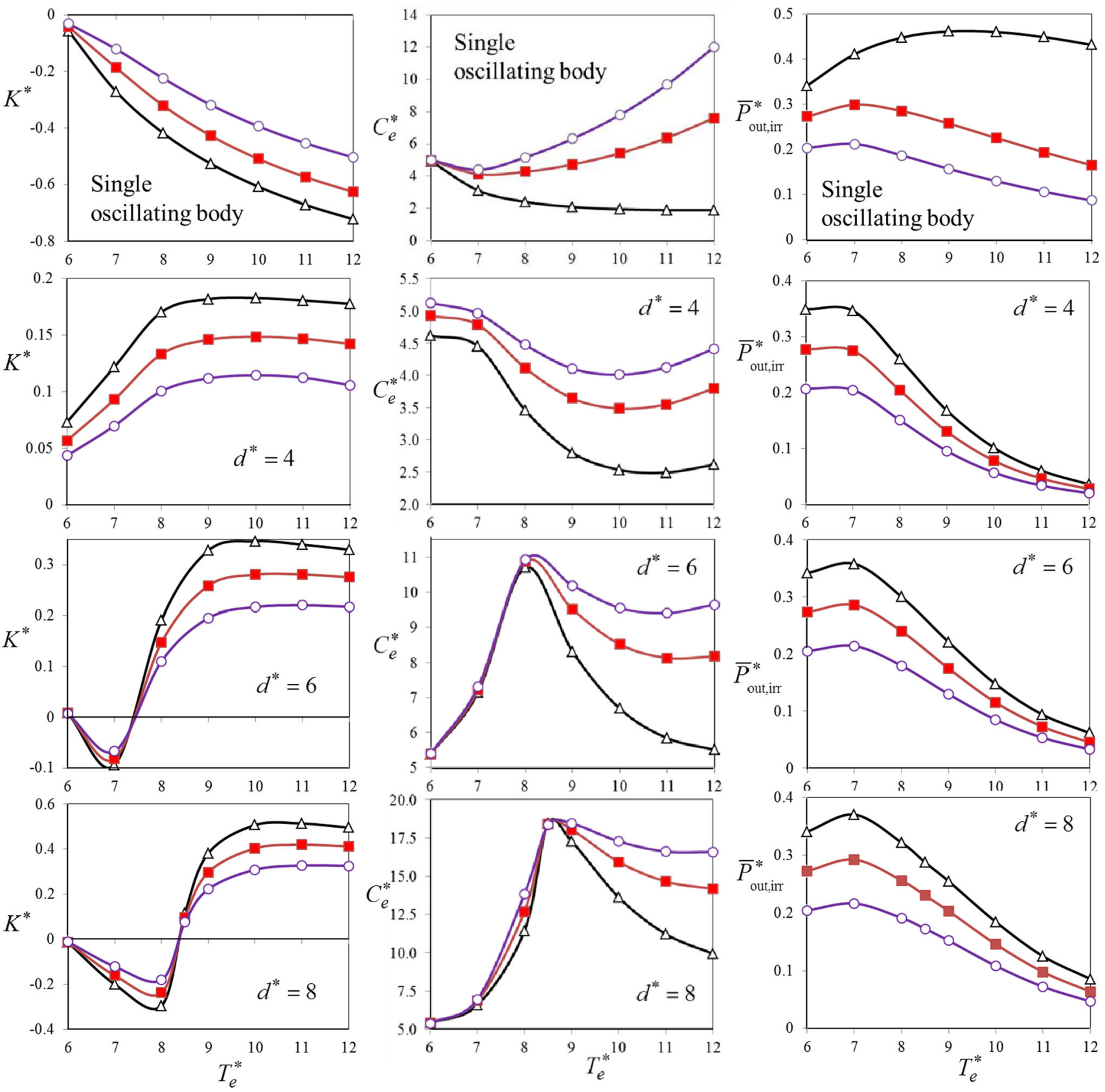

$$
\because \eta=1 \quad \square=0.8 \quad \longrightarrow \eta=0.6
$$

Fig. 6 Dimensionless plot of optimized $K^{*}$ and $C_{e}^{*}$, and maximized power output $\bar{P}_{\text {out,irr }}^{*}$, versus energy period $T_{\mathrm{e}}^{*}$. Plotted curves for $\eta=1.0$ (open triangles), $\eta=0.8$ (closed squares) and $\eta=0.6$

also show that, for fixed $T_{\mathrm{e}}^{*} \cong 7$ (for example $T_{\mathrm{e}}^{*}=7$ ), variations in draught $d^{*}$ may imply quite different optimized values for $K^{*}$ and $C_{e}^{*}$, with negative or positive values for $K^{*}$, depending on $d^{*}$.

The first column of Fig. 6 shows that the three curves of $K^{*}$ versus $T_{\mathrm{e}}^{*}$ cross each other at exactly the ordinate $K^{*}=0$ (this may occur outside the plotted area). It may be seen that the corresponding three curves of $C_{e}^{*}$ versus $T_{\mathrm{e}}^{*}$ (second col- (open circles). The four plots in each column concern, from top to bottom: fixed body 2 (or $d^{*}=\infty$ ), and two oscillating bodies with $d^{*}=4,6,8$

umn of Fig. 6) are tangent to each other at the same abscissae as the ones at which it is $K^{*}=0$. These coincidences may be easily explained. We note first that, if $K=0$, we have pure active power, and $\bar{P}_{\text {out, irr }}$ becomes simply equal to the time-averaged power absorbed from the waves, $\bar{P}_{\text {irr }}$, times $\eta$. Hence, if, for some $T_{\mathrm{e}}$, a pair $\left(K=0, C_{e}\right)$ maximizes $\bar{P}_{\text {irr }}$, then the same pair of values also maximizes $\bar{P}_{\text {out, irr }}$ independently of the efficiency $\eta$. 
Let us assume that the PTO of our two-body heaving device is incapable of providing reactive power. It can still produce as much energy for a value, or a set of values, of $T_{\mathrm{e}}^{*}$ as one that is capable of reactive control. Those are the values of $T_{\mathrm{e}}^{*}$ for which the optimization process yields $K^{*}=0$. We consider the case of draught $d^{*}=8$. Such a value is $T_{\mathrm{e}}^{*}=8.42$, as can be found from the bottom row of Fig. 6 (and the computer program that produced it). The corresponding values are $C_{e}^{*}=17.8$ (independent of $\eta$ ), and $\bar{P}_{\text {out,irr }}^{*}=0.293$ (for $\eta=1.0$ ), $\bar{P}_{\text {out,irr }}^{*}=0.235$ (for $\eta=0.8), \bar{P}_{\text {out }, \text { irr }}^{*}=0.176$ (for $\eta=0.6$ ).

These comments are based on the curves plotted in Fig. 6 from values computed from the approximate Eqs. (30) and (40). The correctness of these values was examined in Appendix 2, where it was found that the relative error $|\varepsilon|$ does not exceed about $1 \%$. Such errors are too small to be noticed in the curves shown in Fig. 6 and do not affect the conclusions drawn above.

It may be of interest to plot the reactiveness ratio introduced in Sect. 2.1, which is redefined here for irregular waves as $G_{\text {irr }}=K\left(\omega_{e} C_{e}\right)^{-1}$, where, as before, $C_{e}=B_{11}\left(\omega_{e}\right) C_{e}^{*}$ and $\omega_{e}=2 \pi / T_{\mathrm{e}}$. We find, for $b=0.4 a$,

$G_{\mathrm{irr}}=\frac{0.02128 T_{\mathrm{e}}^{* 2} K^{*}}{B_{11}^{*}\left(T_{\mathrm{e}}^{*}\right) C_{e}^{*}}$.

We recall that $B_{11}^{*}\left(T_{\mathrm{e}}^{*}\right)$ is represented in Fig. 3 and that the optimized PTO coefficients $K^{*}$ and $C_{e}^{*}$ are plotted in Fig. 6 versus the dimensionless energy period $T_{\mathrm{e}}^{*}$. The optimized reactiveness $G_{\text {irr }}$ is plotted in Fig. 7 versus $T_{\mathrm{e}}^{*}$.

From the definition of $G$ and $G_{\text {irr }}$, we may say that, in a given sea state and for fixed efficiency $\eta$, the ratio of the PTO peak power to the averaged PTO power is expected to increase with $\left|G_{\text {irr }}\right|$. This has important implications in the design and specifications of the PTO equipment. Figure 7 shows that, in optimized conditions, the values of $\left|G_{\text {irr }}\right|$, and consequently of the PTO peak-to-average power ratio, in general increase markedly with the PTO efficiency $\eta$. The variation of $\left|G_{\text {irr }}\right|$ with increasing $T_{\mathrm{e}}^{*}$ (i.e. increasing energy period $T_{\mathrm{e}}$ or decreasing buoy radius $a$ ) depends markedly on the relative draught $d^{*}$. The PTO designer could be looking for zero values for $G_{\text {irr }}$, i.e. no reactive power. Figure 7 shows this to occur for different values of $T_{\mathrm{e}}^{*}$, depending on $d^{*}$ (with a single oscillating body represented by $d^{*}=\infty$ ).

The larger values of $T_{\mathrm{e}}^{*}$ are particularly interesting, since they may represent smaller buoys in typical oceanic sea states. We take, for example, $a=6 \mathrm{~m}$ (buoy diameter $12 \mathrm{~m}$ ) and $T_{\mathrm{e}}=9 \mathrm{~s},\left(T_{\mathrm{e}}^{*}=11.50\right)$, and assume, for the PTO, the relatively good efficiency $\eta=0.8$. We find, for optimal conditions, $\left|G_{\text {irr }}\right|=2.19$ for the single oscillating body, $\left|G_{\text {irr }}\right|=1.00$ for $d^{*}=4,\left|G_{\text {irr }}\right|=0.87$ for $d^{*}=6$ and
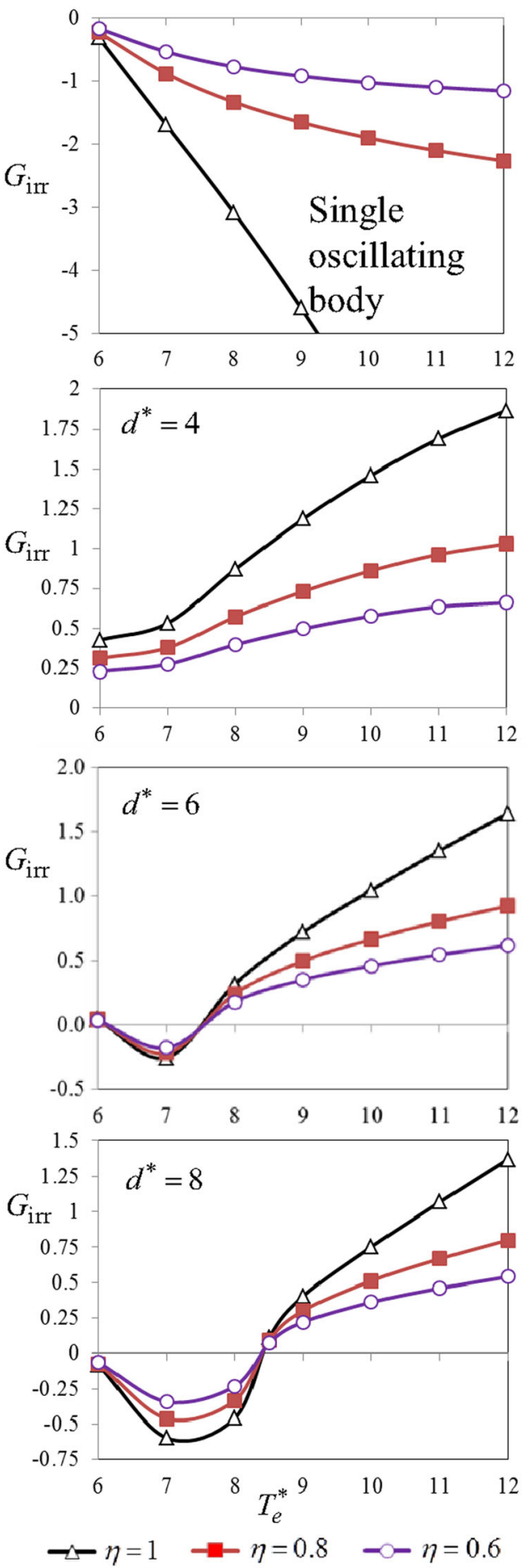

Fig. 7 Reactiveness ratio $G_{\text {irr }}$ versus energy period $T_{\mathrm{e}}^{*}$ for optimized conditions. Plotted curves for $\eta=1.0$ (open triangles), $\eta=0.8$ (closed squares) and $\eta=0.6$ (open circles). The four plots concern, from top to bottom: fixed body 2 (or $d^{*}=\infty$ ), and two oscillating bodies with $d^{*}=4,6,8$

$\left|G_{\text {irr }}\right|=0.74$ for $d^{*}=8$. This shows, in such a case and from the PTO viewpoint, the advantage of the two-oscillatingbody converter over the single-body one. 


\section{Conclusions}

An investigation was carried out on reactive control of an axisymmetric two-body wave energy converter oscillating in heave, particularly on how the optimal PTO impedance is affected by non-ideal PTO efficiency. Regular and irregular waves were considered. The special situation when the inner body is fixed to the sea bottom, that was examined in some detail here, is similar to the single-body single-degree-offreedom converter with a different kind of motion analysed in Strager et al. (2014) and Genest et al. (2014).

The optimization processes showed that, as the PTO efficiency decreases from unity to more realistic values, the reactive part of the PTO impedance should be reduced, while the resistive part should increase. This is in agreement with the results in previous papers on reactively controlled singledegree-of-freedom converters (Strager et al. 2014; Genest et al. 2014).

The major contribution of this paper is the analysis of a two-body two-degree-of-freedom converter. In the case of irregular waves, the draught of the inner body was allowed to take different values, an infinite value being equivalent to fixing the body to the sea bottom (single oscillating body). If the PTO is perfectly efficient, and the case when the inner body is fixed is compared with the case when it is allowed to oscillate, the former appears as clearly superior to the latter, in terms of increased PTO power output, and also because this can be achieved with a device of substantially smaller diameter. However, these advantages tend to vanish as more realistic PTO efficiencies are considered, with maximum values of the dimensionless power output $\bar{P}_{\text {out,irr }}^{*}$ about 0.3 for PTO efficiency $\eta=0.8$, and about 0.21 for $\eta=0.6$. This was found to occur for the dimensionless energy period $T_{\mathrm{e}}^{*} \cong 7$, independently of the draught. However, for fixed $T_{\mathrm{e}}^{*}$, the optimal values of the reactive and resistive parts of the PTO impedance may vary widely depending on the inner body draught. The value of the draught for which the optimized impedance of the PTO is purely resistive may be of special interest. This is relevant when designing a two-body converter of the type considered here and its PTO. The ratio of the PTO peak power to the averaged PTO power is expected to increase with the reactive-to-resistive power ratio, i.e. with the reactiveness ratio $|G|$ or $\left|G_{\text {irr }}\right|$. Besides, larger values of this ratio imply larger peak forces for the same average power level. For given $T_{\mathrm{e}}^{*}$ and efficiency $\eta$, the value of $\left|G_{\text {irr }}\right|$ was found to depend significantly on the draught $d^{*}$. This has important implications in the design and specifications of the PTO equipment. The larger values of $T_{\mathrm{e}}^{*}$ (greater than about 10 ) are particularly interesting, since they may represent relatively small buoys in typical oceanic sea states. In such cases, and from the PTO designer point of view, the two-oscillating-body converter was found to be superior to the single-body one.
Apart from linear water wave theory, several approximations were introduced into the theoretical model developed here. The inner body 2 was assumed long enough for the hydrodynamic interaction between its own bottom and the outer body 1 to be neglected. The error introduced by this approximation is expected to increase with the dimensionless wave period $T^{*}$ or $T_{\mathrm{e}}^{*}$ and with the reciprocal of the dimensionless draught $d^{*-1}$. It may be significant, possibly even for some points plotted in Figs 6 and 7. In such cases, the results should be considered with some care, possibly qualitatively rather than quantitatively. Another approximation is the assumption of a linear PTO and invariant efficiency $\eta$, which are unlikely to be very realistic representations of PTOs of reactively controlled wave energy converters. Such approximations may be acceptable in this kind of investigation that attempts to find trends rather than very realistic values. More realistic modelling should be adopted in the development of particular wave energy converters, possibly involving the computationally much more demanding timedomain analysis.

Acknowledgments This work was funded by the Portuguese Foundation for the Science and Technology (FCT) through IDMEC, under LAETA Pest-OE/EME/LA0022 and contracts PTDC/EMEMFE/103524/2008 and PTDC/EME-MFE/111763/2009, and by Project Offshore Test Station, KIC InnoEnergy, European Institute of Technology. The second author was supported by FCT researcher grant No. IF/01457/2014. The authors want to thank the anonymous reviewers for their constructive comments on the original version of the manuscript.

\section{Appendix 1}

The instantaneous power output from the PTO is $P_{\text {out }}(t)=$ $\eta P(t)$ if $P(t)>0$ and $P_{\text {out }}(t)=\eta^{-1} P(t)$ if $P(t)<0$. Here $\eta \leq 1$ is the PTO instantaneous efficiency that is assumed time-invariant. Analytical expressions for the time-averaged value $\bar{P}_{\text {out }}$ of the power output from the PTO, $P_{\text {out }}(t)$, can be obtained from Eqs. (6) and (7) by integration over one wave period, and are

$$
\begin{aligned}
\bar{P}_{\text {out }}= & \pi^{-1} \omega^{3} C\left|X_{1}-X_{2}\right|^{2}\left[\eta\left(\phi_{3}+\phi_{4}-\phi_{1}-\phi_{2}\right)\right. \\
& \left.+\eta^{-1}\left(\phi_{2}-\phi_{4}\right)\right] \text { if } K \geq 0, \\
\bar{P}_{\text {out }}= & \pi^{-1} \omega^{3} C\left|X_{1}-X_{2}\right|^{2}\left[\eta\left(\phi_{2}+\phi_{3}-\phi_{1}-\phi_{4}\right)\right. \\
& \left.-\eta^{-1}\left(\phi_{2}-\phi_{4}\right)\right] \text { if } K<0,
\end{aligned}
$$

where

$$
\begin{aligned}
& \phi_{1}=\Phi(-\pi / 2 \omega), \\
& \phi_{2}=\Phi(0), \\
& \phi_{3}=\Phi(\pi / 2 \omega), \\
& \phi_{4}=\Phi\left(-\omega^{-1} \arctan G\right),
\end{aligned}
$$


$\Phi(t)=\frac{t}{2}-\frac{1}{4 \omega} \sin (2 \omega t)-\frac{G}{2 \omega} \cos ^{2}(\omega t)$.

Alternative expressions are given in Strager et al. (2014) for a single-degree-of-freedom converter.

\section{Appendix 2}

As mentioned in Sect. 3, Eq. (29) is exactly valid only if $\eta=1$ or if $K=0$. We investigate here the magnitude of the errors if these conditions are not satisfied, especially for the conditions of the points plotted in Fig. 6. To do that, we consider the Pierson-Moskowitz variance density spectrum defined by Eq. (35), and, as in Sect. 4.2, express it in terms of variable $\theta=\left(T_{\mathrm{e}} f\right)^{-1}$, so that $S_{f}(f) \mathrm{d} f=S_{\theta}(\theta) \mathrm{d} \theta$. We find

$S_{\theta}(\theta)=0.1688 H_{\mathrm{s}}^{2} \theta^{3} \exp \left(-0.675 \theta^{4}\right)$

This equation is represented in Fig. 8. To avoid numerical integrations over a very long time period, this continuous spectrum is replaced by a discrete one, concentrated at $\theta_{1}=$ $0.5, \theta_{2}=0.75, \theta_{3}=1, \theta_{4}=1.25$ and $\theta_{5}=1.5$, at intervals $\Delta \theta=0.25$, as shown in Fig. 8. In this way, the instantaneous power output resulting from the superposition of the five components is a periodic time-function of period $\Delta t=15 T_{\mathrm{e}}$.

For this discrete spectrum, the exact value of the timeaveraged power output is

$\bar{P}_{\mathrm{out}, \mathrm{irr}}^{\mathrm{ex}}\left(H_{\mathrm{s}}, T_{\mathrm{e}}\right)=\frac{1}{\Delta t} \int_{0}^{\Delta t} \Phi(t) P_{\mathrm{irr}}(t) \mathrm{d} t$,

where, an in Sect. 3, $\Phi(t)=\eta$ if $P_{\text {irr }}(t) \geq 0$ and $\Phi(t)=\eta^{-1}$ if $P_{\text {irr }}(t)<0$. The instantaneous power $P_{\text {irr }}(t)$ absorbed from the waves is given by Eqs. (22) and (23), with $N=5$ terms in the summations and

$$
A_{w, i}=2 \Delta \theta \times 0.1688 H_{\mathrm{s}}^{2} \theta_{i}^{3} \exp \left[-0.675 \theta_{i}^{4}\right]
$$

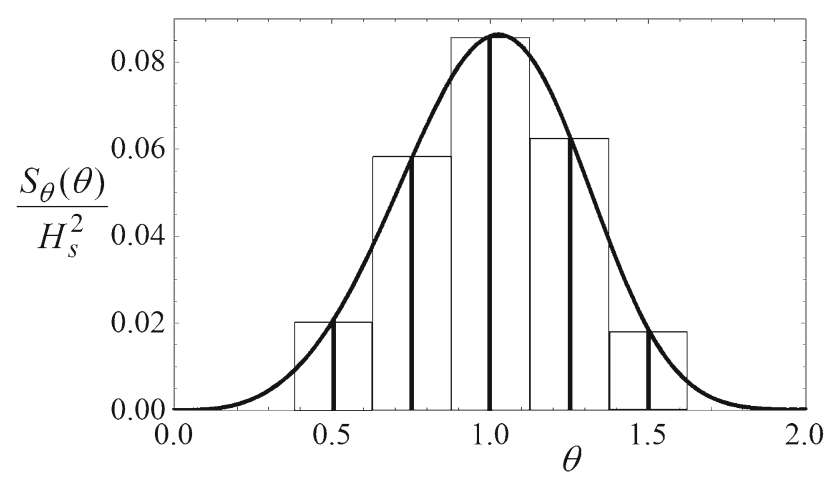

Fig. 8 Discretization of the Pierson-Moskowitz spectrum
Approximate Eq. (30) takes the form

$$
\begin{aligned}
\bar{P}_{\text {out }, \text { irr }}^{\text {app }}\left(H_{\mathrm{s}}, T_{\mathrm{e}}\right) \cong & 2 \Delta \theta \times 0.1688 H_{\mathrm{s}}^{2} \sum_{i=1}^{5} \bar{P}_{\text {out }}^{(1)}\left(\frac{2 \pi}{T_{\mathrm{e}} \theta_{i}}\right) \theta_{i}^{3} \\
& \times \exp \left(-0.675 \theta_{i}^{4}\right)
\end{aligned}
$$

where $\bar{P}_{\text {out }}^{(1)}(\omega)$ is defined, as in Sect. 3, as the time-averaged power output in regular waves of frequency $\omega$ and unit amplitude.

In Eqs. (50) and (52), superscripts "ex" and "app" denote exact and approximate values, respectively. A relative error, defined as

$\varepsilon=\frac{\bar{P}_{\text {out }, \text { irr }}^{\text {app }}\left(H_{\mathrm{s}}, T_{\mathrm{e}}\right)}{\bar{P}_{\text {out }, \text { irr }}^{\mathrm{ex}}\left(H_{\mathrm{s}}, T_{\mathrm{e}}\right)}-1$,

was computed for all combinations $\left(T_{\mathrm{e}}^{*}, K^{*}, C^{*}\right), \eta=$ $0.8,0.6, d^{*}=4,6,8, \infty$ plotted in Fig. 6 , and for several sets of random phases $\alpha_{i}$. In no case was $|\varepsilon|$ found to exceed 0.01 . These values concern the approximation provided by Eq. (52) based on a discrete spectrum. It seems reasonable to conclude that, in the case of the continuous Pierson-Moskowitz spectrum adopted in Sect. 4, the relative errors are of the same order of magnitude, and that the approximation provided by Eqs. (30) and (40) is acceptable within the framework of the paper.

\section{References}

Elwood D, Schacher A, Rhinefrank K, Prudell J, Yim S, Amon E et al. (2009) Numerical modelling and ocean testing of a direct-drive wave energy device utilizing a permanent magnet linear generator for power take-off. In: Proceedings of the 28th international conference ocean offshore arctic engineering, ASME. Honolulu. Paper No. OMAE2009-79146

Evans DV (1976) A theory for wave-power absorption by oscillating bodies. J Fluid Mech 77:1-25

Evans DV (1980) Some analytic results for two and three dimensional wave-energy absorbers. In: Count B (ed) Power from sea waves. Academic Press, London, pp 213-249

Falcão AF de O (2010) Wave energy utilization: a review of the technologies. Renew Sustain Energy Rev 14:899-918

Falnes J, Budal K (1978) Wave power conversion by point absorbers. Nor Marit Res 6:2-11

Falnes J (2002a) Optimum control of oscillation of wave-energy converters. Int J Offshore Polar Eng 12:147-155

Falnes J (2002b) Ocean waves and oscillating systems. Cambridge University Press, Cambridge

Falnes J (2007) A review of wave-energy extraction. Mar Struct 20:185201

Gato LMC, Falcão AF de O (1989) Aerodynamics of the Wells turbine: control by swinging rotor blades. Int J Mech Sci 31:425-434

Gato LMC, Eça LRC, de Falcão AF de O (1991) Performance of the Wells turbine with variable pitch rotor blades. J Energy Resour Technol Trans ASME 113:141-146 
Genest R, Bonnefoy F, Clément AH, Babarit A (2014) Effect of nonideal power take-off on the energy absorption of a reactively controlled one degree of freedom wave energy converter. Appl Ocean Res 48:236-243

Goda Y (2002) Random seas and design of maritime structures, 2nd edn. World Scientific, Singapore

Hansen RH, Kramer M (2011) Modelling and control of the Wavestar prototype. In: Proceedings of the 9th European wave tidal conference. Southampton

Holthuijsen LH (2008) Waves in oceanic and coastal waters. Cambridge University Press, Cambridge

Korde UA (2003) Systems of reactively loaded coupled oscillating bodies in wave energy conversion. Appl Ocean Res 25:79-91

López I, Andreu J, Ceballos S, Martinez de Alegria I, Kortabarria I (2013) Review of wave energy technologies and the necessary power-equipment. Renew Sustain Energ Rev 27:413-434

McKay EBL (2012) Resource assessment for wave energy. In: Sayigh A (ed) Comprehensive renewable energy, vol 8. Ocean energy. Elsevier, Amsterdam, pp 11-77

Perdigão J, Sarmento A (2003) Overall-efficiency optimisation in OWC devices. Appl Ocean Res 25:157-166

Ricci P, Lopez J, Santos M, Ruiz-Minguela P, Villate JL, Salcedo F, Falcão AF de O (2011) Control strategies for a wave energy converter connected to a hydraulic power take-off. IET Renew Power Gener 5:234-244

Salter SH, Jeffery DC, Taylor JRM (1976) The architecture of nodding duck wave power generators. Nav Archit 1:21-24
Sarmento AJNA, Gato LMC, Falcão AF de O (1990) Turbine-controlled wave energy absorption by oscillating water column device. Ocean Eng 17:481-497

Strager T, Fernández-López P, Vidal Sanchez E, Neuville AM, Giorgio G, Murtesan T, Andersen P et al (2014) Optimizing reactive control in non-ideal efficiency wave energy converters. In: Proceedings of the 33rd international conference ocean offshore arctic engineering, ASME. San Francisco. Paper No. OMAE2014-23005

Taylor JRM, Caldwell NJ (1998) Design and construction of the variable-pitch air turbine for the Azores wave energy plant. In: Proceedings of the 3rd European wave energy conference. Patras, pp 328-337

Tedeschi E, Carraro M, Molinas M, Mattavelli P (2011) Effect of control strategies and power take-off efficiency on the power capture from sea waves. IEEE Trans Energy Convers 26:1088-1098

Vidal Sánchez E, Hansen RH, Kramer MM (2012) Early performance assessment of the electrical output of Wavestar's prototype. In: Proceedings of the 4th international conference ocean energy. Dublin

Vidal Sánchez E, Hansen RH, Kramer MM (2014) Control performance assessment and design of optimal control to harvest ocean energy. IEEE J Ocean Eng. (To be published)

Weber J, Mouwen F, Parrish A, Robertson D (2009) Wavebob-research $\&$ development network and tools in the context of systems engineering. In: Proceedings of the 8th European wave tidal energy conference. Uppsala, pp 416-420 